\title{
Le siècle de Sartre ou quand la foi n'était jamais la bonne
}

\section{Camille Riquier}

\section{(2) OpenEdition}

1 Journals

Édition électronique

URL : https://journals.openedition.org/alter/2245

DOI : $10.4000 /$ alter.2245

ISSN : 2558-7927

Éditeur :

Association ALTER, Archives Husserl (CNRS-UMR 8547)

\section{Édition imprimée}

Date de publication : 1 novembre 2020

ISBN : 978-2-9550449-6-4

ISSN : 1249-8947

\section{Référence électronique}

Camille Riquier, «Le siècle de Sartre ou quand la foi n'était jamais la bonne », Alter [En ligne], 28 | 2020, mis en ligne le 22 décembre 2020, consulté le 12 décembre 2021. URL : http:// journals.openedition.org/alter/2245 ; DOI : https://doi.org/10.4000/alter.2245

Ce document a été généré automatiquement le 12 décembre 2021.

Revue Alter 


\title{
Le siècle de Sartre ou quand la foi n'était jamais la bonne
}

\author{
Camille Riquier
}

Tantôt nous retenons que Dieu s'est donné au temps de sa venue, mais nous pensons que le don,

le message et la vie du Christ sont épuisés. [...]

Tantôt, en sens inverse, nous oublions que Dieu s'est donné en personne, absolument, pour croire que tout est à inventer, que l'Église n'a pas d'héritage ${ }^{1}$.

1 Sartre nous offre, semble-t-il, les moyens de bien poser le problème de notre incrédulité nouvelle, pour peu qu'on n'appauvrisse pas le sens qu'il a donné à la notion de «mauvaise foi ». Gardons-nous en effet de la maintenir au niveau d'analyse où luimême s'était placé quand il décrivait les atermoiements de la coquette, qu'on a depuis considérée abusivement comme le parangon de la fausse vertu. Bien sûr, la signification que Sartre confère à la " mauvaise foi » est très large, puisqu'elle s'étend à toutes les dimensions de l'existence humaine et frappe le moindre de nos gestes d'un sceau d'inauthenticité. Mais reconnaissons que la notion gagne en pertinence quand elle s'applique à la sphère plus étroite du religieux, où elle puise son premier emploi. Elle doit également y gagner en compréhension, puisque son acception profane ne semble pas pouvoir s'en affranchir absolument. N'a-t-il pas fallu que la mauvaise foi y conservât quelques attaches pour opérer avec une telle efficacité partout désormais où Dieu n'est plus, c'est-à-dire partout? Il faut en effet se demander quelle est plus spécifiquement cette foi qui a été définitivement sapée au point d'avoir été rendue mauvaise dans son essence jusqu'à ruiner en l'homme la totalité de ses croyances. Telle est l'interrogation qui doit guider notre lecture de Sartre, puisque le soupçon y est fort à tout le moins que la mauvaise foi n'a pu se déployer et prendre des proportions si vastes que depuis l'arrière-fond d'un Dieu manquant.

2 Le monde a-t-il vacillé ? Ne reste-t-il plus une seule évidence à laquelle adhérer sans réserve ? La science n'est-elle désormais qu'un moyen de s'évader vers le savoir, faute 
d'être le savoir véritable? Nul doute que pour Sartre une distance nous a irrémédiablement séparé de l'être, à ce point que nous n'y sommes plus assurés de rien. Pourtant, gisant au milieu des choses, dissimulée au fond de toutes les anciennes évidences en train de s'évanouir, une certitude demeure, sur laquelle Sartre n'est jamais revenu, ni même venu, qu'il ne montre, ni ne démontre: Dieu n'existe pas. À douze ans, dans une rue de La Rochelle, il s'était posé la question et estima l'avoir réglée " une fois pour toutes » : «Ce fut une évidence authentique, encore que je ne sache absolument plus sur quoi elle s'appuyait ${ }^{2}$.» Définitivement acquise à l'adolescence, laissée là où elle est, elle ne s'appuie sur rien, mais tout s'appuie sur elle. Retenue à la marge, au seuil de l'œuvre, elle est l'absence qu'il n'y a plus à penser mais dont l'impensé donne à Sartre le pouvoir de penser l'évanescence de toutes choses.

3 Alors peut-être que la coquette est de mauvaise foi dans la mesure où elle s'ingénie à recevoir de son amant sans avoir à lui donner en retour, l'air de rien. Là est en effet le secret artifice qu'elle pare d'un semblant de vertu. Mais de la profondeur de quelles racines et à quelles tout autres conséquences n'est-on pas conduit si l'on prend pour fil conducteur la figure moderne du croyant, dont la foi est d'emblée disqualifiée par la mauvaise foi qui lui serait inhérente. Ce n'est pas le mensonge que l'on se fait à soimême à quoi l'on doit conclure, mais rien de moins selon Sartre que l'immense mystification opérée par la religion, coupable d'avoir coupé l'homme de lui-même en logeant sa liberté dans la volonté d'un Autre. Dans l'optique adoptée ici, la "mauvaise foi » doit surtout servir à décrire la situation particulière que la modernité a faite au christianisme, en mettant les athées comme les chrétiens dans la nécessité d'entretenir leur foi dans un monde privé de transcendance, reposant sur l'unique "évidence authentique » dont ils disposent, que Dieu n'existe pas.

Sartre ne nous autorise pas seulement à douter de la sincérité de ceux qui aujourd'hui recouvrent la foi après y avoir renoncé. Il laisse grandir le soupçon qu'il ne se rencontre aujourd'hui plus une seule foi véritable. Seuls les piliers de Notre-Dame tiennent encore debout. S'«il est des époques où l'incroyance ne peut être que verbale $»^{3}$, son époque aura été l'une des premières où la foi ne fut jamais que de bouche et professée seulement, mais non plus ressentie par le cœur. Elle a introduit le croyant lui-même dans une comédie désespérée du croire, dans laquelle il est astreint à jouer le double rôle du dupeur et du dupé. Sa foi y est condamnée à n'être jamais la bonne, rien d'autre dans son essence qu'une mauvaise foi. Tout se passe désormais comme si la foi et la bonne foi s'excluaient mutuellement et que le fidèle ne pouvait croire à ce qu'il croit sans s'accommoder d'un certain aveuglement et, à la longue, d'une discrète complaisance à soi. En un sens, telle était déjà la difficulté qu'il avait été donné à André Gide de formuler. À ses amis catholiques qui lui adjuraient de revenir au catholicisme, à Paul Claudel en particulier, le bien nommé "rouleur convertisseur », il devait d'abord répondre : «la foi tout court ne dispense pas de la bonne ». Il hésite ensuite, différa jusqu'à juger enfin, réflexion faite, qu'elles étaient rigoureusement incompatibles : « La bonne foi est une vertu essentiellement laïque, que remplace la foi tout court $»^{4}$.

5 Cependant, la question demeure de savoir si l'expérience humaine de la foi, en violente torsion sur elle-même dans la situation d'être-sans-Dieu où elle opère, s'y était alors dégradée en inévitable mauvaise foi, ou si, au contraire, comme Sartre l'a d'abord soutenu, elle y a trouvé les conditions propices à sa révélation authentique, en sorte qu'en elle se trahirait la «mauvaise foi » qu'elle avait depuis toujours été. Dans la 
mesure où la réalité humaine a un rapport trans-historique à son être, Sartre a en effet pu accorder à l'historicité de son époque le privilège insigne d'avoir mis celui-ci à nu : de même que son être-en-guerre, par sa radicalité, lui donne à voir l'être-au-monde de la réalité-humaine, de même, son être-sans-Dieu doit lui révéler la mauvaise foi qui a toujours été la sienne, en raison de l'absurdité manifeste dans laquelle tombe le chrétien aujourd'hui, puisqu'il prie un Dieu qu'il sait sourdement ne pas exister. C'est ce qu'il nous faut contester, en y reconnaissant l'illusion rétrospective à laquelle cède trop souvent le philosophe pour qui la vérité singulière qu'il a été en droit de conclure de l'examen de son temps doit recevoir après coup un coefficient d'universalité et de nécessité qui lui permet d'avoir été vraie depuis toujours.

6 La notion de « mauvaise foi » éclaire de sa lumière crue et implacable la situation que la modernité a faite à la croyance, mais un doute subsiste qui doit nous retenir de lui accorder également la puissance de révéler abstraitement l'être de l'homme, indépendamment de la forme singulière que les circonstances historiques l'ont contraint d'épouser. Il est dès lors préférable de procéder ici par étapes, faute d'être assuré de la cohérence générale de l'analyse menée par Sartre. Et s'il s'avère que celleci n'a pu gagner en extension, et s'appliquer à tous les âges de la foi, qu'au détriment de sa valeur descriptive, il sera alors toujours possible d'en rabattre et de se maintenir dans la sphère où celle-ci retrouve toute sa pertinence, c'est-à-dire en limitant sa portée à l'époque moderne qu'elle a pour fonction de ressaisir dans son universalité singulière.

7 C'est ainsi. Sartre est un homme du $\mathrm{xx}^{\mathrm{e}}$ siècle. Il est né après la «mort de Dieu ». Son existence est enregistrée, inscrite en son lieu et à sa date. Elle est relative à sa propre historicité. Et sa pensée, objectivée dans ses œuvres, est elle-même située. Il faut donc travailler Sartre, en partie du moins, comme il travaillait ses auteurs. Il doit être étudié en tant qu'il est également un " universel singulier : totalisé et, par là même, universalisé par son époque, il la retotalise en se reproduisant en elle comme singularités $»$. En partie seulement, car notre intention est moins de fixer la singularité de l'œuvre de Sartre que de surprendre dans son reflet l'universalité de son époque, elle-même considérée sous l'angle déterminé de sa foi et de son doute.

\section{Une eidétique de la mauvaise foi}

8 Il convient en premier lieu de se reporter à L'Être et le Néant et à la description d'essence que Sartre y propose de la "mauvaise foi ». Dans la mesure où celle-ci constitue le mode d'être inauthentique qui a toujours été celui de la réalité-humaine et qu'elle existe au fond de toute foi, elle sert à caractériser la subjectivité dans son multiple rapport au monde, à soi-même, à Autrui ou à Dieu. Autrement dit, elle doit s'appliquer à la forme générale prise par la croyance, dont la foi religieuse semble n'être ici qu'une des déclinaisons possibles. Or, si la foi est par essence "mauvaise foi », c'est qu'elle porte la contradiction dans l'acte même par lequel elle se constitue. Car la foi n'est jamais triomphante, pleine et entière. Et il suffit d'expliciter sa croyance, serait-ce simplement d'en prendre conscience, pour la savoir d'emblée en déficit d'elle-même, dans la mesure où elle ne possède pas intuitivement l'objet de sa croyance.

Autrement dit, la foi ne peut poser son objet et se ressaisir comme conscience-decroyance sans vaciller et menacer de se détruire. Visant un être qui lui échappe par principe, elle ne parvient à l'atteindre tout de même qu'à la condition de l'affecter d'un 
certain coefficient de non-être. Pour cette raison, la croyance croit moins qu'elle ne veut croire à ce qu'elle croit, tout en sachant secrètement qu'elle n'y croit pas, puisqu'elle ne fait qu'y croire et qu'au besoin il lui faut elle-même s'en persuader : « au moment où je veux me croire courageux, je sais que je suis lâche». Telle est la contradiction interne qui grève la foi dès son commencement : pour peu qu'on y prête attention, "croire, c'est ne pas croire ${ }^{6}$ ". Partout et toujours, il ne saurait exister quelque chose de tel qu'une lucidité de la foi. Doit-on s'y résoudre et en prendre son parti? Mais celui qui décide de ne plus croire ne peut davantage en former le clair projet sans se contredire, puisqu'il sait alors qu'inversement «ne pas croire, c'est croire ». Pas plus qu'il n'avait cru de bonne foi, il ne peut désormais choisir en conscience d'être de mauvaise foi. D'un côté, celui qui veut croire de façon résolue ne se dissimulera pas longtemps qu'il croit " pour ne pas croire», pour la raison qu'il sait sourdement ne pas croire à ce qu'il croit; et c'est pourquoi le fanatique cache mal le fait qu'il croit par désespoir d'avoir perdu la foi ; mais de l'autre côté, celui qui se résigne à ne jamais croire finira peut-être par s'avouer qu'il ne croit pas " pour croire ", pour la raison symétriquement inverse qu'il tient encore secrètement à ce qu'il a échoué à croire; et c'est pourquoi l'homme cynique n'est qu'un idéaliste déçu qui continue à croire à ce qu'il nie par la manière même dont il le nie. Bref, en toute rigueur, la foi est impossible; et il n'y a pas même la mauvaise dont nous puissions conclure qu'elle est la bonne.

Mais le plus étonnant n'est pas encore dit. Il est dans ce qui suit : la mauvaise foi est peut-être impossible; pourtant elle est réelle. Elle ne peut exister, pas plus que la bonne foi ; mais son fait n'en est pas moins irrécusable. Et c'est ainsi que nous croyons. Il faut donc qu'elle soit autre chose que le cynisme et qu'elle puisse elle-même se nier comme mauvaise foi. Pour l'expliquer, Sartre remonte jusqu'aux conditions qui l'ont rendue possible au sein de la conscience. En sorte que « le projet d'être de mauvaise foi doit être lui-même de mauvaise foi ", en raison de la structure interne de la conscience dont le mode d'être est de ne pas être ce qu'elle est et d'être ce qu'elle n'est pas. On comprend alors que la conscience n'a pas besoin pour croire de trop réfléchir; il lui faudrait même, si cela était possible, accompagner sa croyance de façon seulement diffuse et spontanée. Car la conscience-croyante ne peut qu'éprouver son propre embarras à croire. Pour ne pas souffrir d'être prise en défaut chaque fois qu'elle s'y efforce, elle doit prendre la résolution tacite "de ne pas trop demander, de se tenir satisfaite quand elle sera mal persuadée, de forcer par décision ses adhésions à des vérités incertaines ${ }^{7}$. Par-là, elle s'affecte de mauvaise foi, en acceptant sa propre " incapacité à croire ». Elle se dit que "de toute façon je ne suis pas un saint ", qu'après tout « même les saints ont eu des doutes ", que personne n'est jamais assez croyante et qu'il lui est impossible de satisfaire l'idéal de plénitude qu'elle réclame. Sachant confusément qu'un néant toujours la sépare de l'être auquel elle aspire, elle se résigne donc à réviser ses exigences à la baisse et à ne pas être pleinement convaincue quand elle croit. Bref, elle transforme l'impossibilité de la croyance comme la seule manière pour elle de croire : « je définirai comme ma croyance cette croyance impossible ».

11 C'est ainsi que la mauvaise foi s'introduit dans la foi et la gouverne en sous-main, en utilisant «l'autodestruction du fait de conscience » pour assurer paradoxalement son maintien. Elle vit de cela même qui la tue en s'appuyant sur ses propres conditions d'impossibilité. Dans la mesure où « croire, c'est ne pas croire ", on ne réussit à croire, et à se laisser gagner par "l'évidence non persuasive ", qu'en étant de mauvaise foi. Et pour ce faire, il suffit d'accepter de n'avoir qu'une foi mauvaise, qu'on sait secrètement 
n'être pas la foi qu'elle est (ou prétend être). En d'autres termes, pour que la conscience soit disposée à croire ce qu'elle ne croit pas, il faut au préalable qu'elle soit déterminée à ne pas croire ce qu'elle croit, en se situant en-deçà d'une foi pleine et entière. En sorte que la croyance "ne peut exister que comme troublée ", et cela dès l'origine, avant même qu'elle n'ait à poser son objet dans la réflexion. C'est dire que rien n'explique ce trouble, puisqu'ici « rien ne peut séparer la conscience (de) croyance de la croyance »: "la croyance n'est rien d'autre que la conscience (de) croyance» et celle-ci ne se distingue pas davantage de celle-là, puisqu'elle «est pour faire l'acte de foi ». Si donc une fissure les sépare, qui altère irrémédiablement la croyance, la foi ne peut résider pour Sartre que dans la «distance nulle» (entre la conscience et la conscience (de) croyance) que l'être de la conscience « porte dans son être » en tant qu'il est son propre néant. Mieux, elle est le Néant; et «nulle part nous ne pourrions le saisir dans une pareille pureté ». En effet, la croyance n'est pas: en surgissant du sein même de la conscience, elle "est sa propre décompression d'être ", un néant qui est aussi "son néant ». Elle est cette fissure par laquelle la mauvaise foi projette de s'échapper de l'en soi, quitte à l'agrandir en béance, en voulant la " désagrégation intime $»^{8}$ de l'être qu'on est. Bref, en toute rigueur, la foi n'existe pas sans que le néant toujours ne s'y retrouve de part et d'autre; et il n'y a pas jusqu'à la bonne foi dont nous ne puissions conclure qu'elle est toujours mauvaise.

Si des réticences nous empêchent d'accompagner Sartre jusque dans ses conclusions, la critique à laquelle il nous invite porte cependant moins sur l'analyse qu'il propose de la "mauvaise foi » que sur le champ d'application qu'il lui demande de couvrir. En voulant celui-ci aussi vaste en surface que profond par les racines que la mauvaise foi y plonge, il s'est autorisé à déduire de la possibilité pour l'homme d'être de mauvaise foi rien de moins que la structure de son être et l'existence inauthentique à laquelle sa liberté s'est enchaînée comme à un destin sans issue. Or, à vouloir que tous les hommes soient des comédiens, on s'expose au risque que plus personne ne le soit; et par excès de lucidité, on se prive du vrai discernement et d'une critique féconde. "Comment

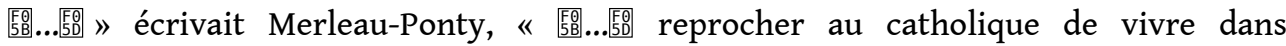
l'équivoque si tout le monde y vit et si la mauvaise foi est l'essence même de la conscience $"{ }^{9}$ ? Le reproche est d'ailleurs similaire à celui que Merleau-Ponty avait parallèlement formulé contre la liberté sartrienne, annulée par son excès même d'absolu, puisqu'on la retrouvait égale dans toutes nos actions, que l'esclave vive dans la crainte ou qu'il brise ses chaînes. Dire de la mauvaise foi qu'elle est partout, n'est-ce pas dire aussi bien qu'elle n'est nulle part ? Les chrétiens ne méritent plus qu'on les en accuse si tout le monde est de mauvaise foi, et les autres pas moins qu'eux. Leur foi vaut toutes les autres. Il n'est pas même possible de la perdre, puisqu'elle n'a jamais été gagnée. Le fou, le saint, l'enfant, tous alors seraient des comédiens, autant qu'ils sont, avec dans le cœur, rongés par la conscience de leur propre néant, le poison de la mauvaise foi. Faudra-t-il se résoudre à ce que l'être soit définitivement " pourri par luimême»?

On sait que Sartre lui-même ne s'y était pas résigné et que la morale authentique qu'il projetait d'écrire devait permettre d'« échapper radicalement à la mauvaise foi $»^{10}$. S'il a plusieurs fois varié sur ce qu'il entendait poursuivre à travers la notion d'«authenticité », elle devait surmonter la fausse alternative de la bonne et de la mauvaise foi. Dès 1939, il avait fixé la hauteur à laquelle une telle foi authentique devait se tenir afin que l'homme puisse "réaliser pleinement son être-en-situation »: une « fidélité à soi » qui est également une «fidélité au monde », c'est-à-dire une façon de 
répondre à ce qu'exige la situation en se déterminant "soi-même à "être-pour" cette situation $»^{11}$. On sait aussi que Sartre n'est pas parvenu à élever sa morale au niveau de l'idéal projeté et qu'au couronnement de l'œuvre, il y a Les Mots et l'aveu de son propre échec, l'échec auquel il se rendît compte après coup que son projet d'écriture devait inévitablement être conduit.

\section{La mauvaise foi ou la situation qui a été faite à la foi au $\mathrm{XX}^{\mathrm{e}}$ siècle}

Peut-être est-il alors préférable de restreindre la portée de l'analyse, si nous voulons, avec les ajustements correspondants, nous resserrer sur la part de vérité qu'elle contient. Sartre avait jeté dans ses Carnets de la drôle de guerre les premières idées qui devaient servir à la composition de L'Être et le Néant. Rédigées durant sa période de mobilisation, entre 1939 et 1940, elles étaient d'ailleurs plus nettement marquées par la situation qu'il lui avait été faite à lui et à ses contemporains. Et lorsqu'en 1954-1955, il revient sur ses anciennes notes dans le Cahier Lutèce, il semble endosser le reproche qui lui avait été adressé : dans la mesure où " chaque époque a une universalité de sa foi et de son doute ", c'est bel et bien à partir de la sienne, singulièrement imprégnée par la " mort de Dieu ", qu'il peut dire : " personne ne croit ». Ce qu'il décrivait touchait juste, mais devait se comprendre depuis la situation objective qu'un tel événement avait inévitablement faite à l'athée comme au chrétien. Resserrée de la sorte, on s'aperçoit alors que la notion de mauvaise foi, que Sartre avait d'abord développée pour ellemême, relève éminemment du monde moderne déchristianisé, voire inchrétien, en lequel elle a trouvé l'occasion de déployer tous ses ressorts.

Sa charge critique semble trouver son exacte portée sitôt qu'on quitte le plan eidétique de l'analyse pour le plan historique, en la replaçant dans l'époque qui la lui avait révélée. On est alors invité à reprendre sa description dans le prolongement d'une étude inachevée que Sartre avait commencé d'écrire en 1952 sur Mallarmé. Son premier chapitre a pour titre "Les héritiers de l'athéisme» et Sartre y décrit le désespoir des poètes symbolistes du XIX $x^{e}$ siècle, à qui on a supprimé les deux thèmes que la poésie avait toujours chéris : l'homme et Dieu. Nés dans une société sans Dieu, " on les a faits athées, ils ont subi la déchristianisation avant d'avoir l'âge de décider par eux-mêmes ». Quels moyens auront ces héritiers alors de retrouver la foi, puisque leurs parents l'ont perdue pour eux jusqu'à les faire ce qu'ils sont :

Essaieront-ils de retrouver les croyances perdues? Mais ce serait revenir en arrière.

Ils savent bien, au fond de leur cœur, que le passé ne se recommence pas. Il peut

leur arriver de faire semblant de croire, pour les besoins de la cause. Mais ils n'ignorent pas que leur foi est de mauvaise foi ${ }^{12}$

L'événement de la "mort de Dieu » décide d'un avant et d'un après, en deçà duquel il leur est impossible de remonter. "Si la foi se perd, l'athéisme ne peut se perdre ». Les enfants veulent-ils revenir à la foi, toujours «ils retiennent en eux l'incroyance qu'ils prétendent dépasser ». L'athéisme a été une négation de la foi ; en sorte qu'après lui, «à partir de 1850 , la foi est une négation de la négation ${ }^{13}$. Aujourd'hui encore, nous sommes les héritiers de cette aporie, et tous ceux qui sont tentés de croire ne peuvent se rapporter à leurs croyances que sur fond de l'évidence reçue que Dieu n'existe pas.

Autrement dit, nous ne voyons pas qu'avant cette date il faille supposer que les hommes aient toujours été de mauvaise foi. Et Sartre ne s'était auparavant autorisé, 
semble-t-il, à en faire sa conclusion qu'au prix d'une équivoque. Car de deux choses l'une: ou bien la mauvaise foi présente "une forme autonome et durable ", voire «implique un style de vie constant et particulier », et Sartre est en droit de l'appliquer à tous les âges de la foi ; ou bien son existence est «fort précaire » et "il est évident qu'elle oscille perpétuellement entre la bonne foi et le cynisme ${ }^{14}$. Et alors la foi ne peut se prolonger indéfiniment elle-même sur d'aussi longues périodes, sans trahir l'artifice sur lequel elle repose. Elle ne peut convenir qu'à l'étrangeté de son siècle, que Sartre, mieux que d'autres, a réussi à ressaisir dans toute son étrangeté :

En 1930, les athées croient que Dieu est mort comme personne mais que ses vertus sont éparses dans le monde. Les chrétiens croient qu'ils croient à l'existence de Dieu mais ils croient à la réalité de son inexistence (c.-à-d. que c'est le Néant de Dieu qui est Dieu); au lieu que pour l'athée, c'est l'attribut de Dieu qui existe seul. Le chrétien nomme Dieu un rien et l'athée ne nomme pas Dieu qui est l'Être. Telle était mon époque ${ }^{15}$.

18 C'est bel et bien de son époque, marquée singulièrement, que Sartre peut dire en effet : «personne ne croit ». Ce qu'il avait décrit touchait juste mais valait d'abord, surtout, depuis la situation objective dans laquelle la mort de Dieu avait fini par inscrire l'athée comme le chrétien, rongeant leur foi, la pénétrant de néant: pour l'athée, Dieu est l'Être et c'est pourquoi il n'y croit pas; pour le chrétien, Dieu est Néant et c'est pourquoi, tout en croyant croire, il n'y croit pas non plus.

Dans ce siècle meurtri par la violence et la guerre, il était donc bien difficile de reconnaître les siens et de savoir du chrétien ou de l'athée lequel a finalement été le plus croyant des deux. Si les chrétiens voulaient croire en Dieu, cela devait être « malgré son Absence - les malins diront : à cause d'elle ${ }^{16}$. Une théologie de « la mort de Dieu » s'est ainsi efforcée de surmonter le désastre de l'événement : puisque Dieu est l'Être et que l'athée nie la foi, il faut nier cette négation pour maintenir sa foi et donc croire à Dieu comme Néant, à l'Absence de Dieu comme la réalité même de sa présence, bref comme un "Dieu sans l'Être ». Inutile de citer les noms; ils sont nombreux, parmi lesquels Dietrich Bonhoeffer et Simone Weil. La situation faite aux athées n'était pas moins artificielle et sinueuse. Car s'ils voulaient croire en quelque chose, c'était en dépit de leur certitude qu'il n'y avait pas de Dieu. Et ils n'y parvinrent qu'en escamotant la contradiction qui le leur permettait: nier l'existence de Dieu et simultanément défendre, avec une même fougue, les valeurs absolues qui avaient appartenu à son essence avant d'en avoir été décrochées : l'Être, la Vérité, la Morale, la Justice, la Liberté, etc. On se trouve devant un paradoxe inouï, qui prend d'ailleurs le contre-pied exact de celui que saint Jacques portait au compte de la foi démoniaque : « Toi, tu crois qu'il y a un seul Dieu ? Tu fais bien. Les démons le croient aussi, et ils tremblent ${ }^{17}$. Si donc les démons ne croient pas en Dieu (credere in Deum), encore qu'ils croient que Dieu existe (credere Deum), ici, c'est l'inverse: les athées ne croient pas que Dieu existe (credere Deum), encore qu'ils continuent à croire en ses attributs (credere in Deum). Nul doute d'ailleurs que le Bien, le Juste, le Vrai soient demeurés vivants dans le cœur d'un grand nombre d'entre eux, mais parce que leur foi était compromise, ils s'étaient mis dans la peine de s'y reporter comme à des absolus, ne pouvant s'y accrocher sans mauvaise foi. C'est ainsi le temps des fois dévoyées et des espérances aventureuses. Et après tout, qu'importe, ajoute Merleau-Ponty, celles-ci étaient peut-être préférables à la foi équivoque du christianisme et du catholicisme, incessamment tiraillés entre la «religion du Père» («Dieu intérieur ») et la « religion du Fils» («Dieu extérieur »). Peut-être était-il temps que l'une et l'autre cèdent la place à la "religion de l'Esprit » 
dans laquelle elles pourraient se réconcilier. Et l'Esprit souffle où il veut. Certes, en choisissant d'adhérer à une idéologie et d'engager leur foi dans un parti politique plutôt que dans une Église, les hommes du $\mathrm{xx}^{\mathrm{e}}$ siècle n'ont pas manqué de se jeter dans d'autres contradictions, aux conséquences parfois dramatiques. Mais qui savait alors? Qui sait d'ailleurs encore maintenant si tout y était mauvais : «Il y a peut-être des prières qui sont mal adressées et qui arrivent, bien, tout de même $»^{18}$.

Il n'empêche qu'après la "mort de Dieu », la foi ne pouvait lui survivre sans que la mauvaise foi ne la pénétrât. Celle-ci n'avait pas remplacé celle-là, mais elle lui était nécessaire pour s'accorder le sursis qu'elle réclamait. Sartre n'y échappa pas non plus, également prisonnier de ce qu'il dénonçait. En cela encore, il continue de s'inscrire dans les limites historiques de son époque. Car la mauvaise foi est bien d'abord le problème que Sartre s'était posé à lui-même. La révolte permanente qui a défini son geste de pensée devait coexister en lui avec la crainte sans cesse renaissante d'avoir été sa propre dupe. Dans Les Mots, l'homme de cinquante ans se découvre ultimement mystifié par l'enfant oublié qu'il fut et duquel il avait reçu la foi en sa propre vocation d'écrivain : une foi invincible dans son génie littéraire, avec la conviction qu'une tombe l'attendait au Père-Lachaise, peut-être au Panthéon, et en même temps, dans le cœur, "le soupçon de son inconsistance ». Sartre avait peut-être "perdu la foi à douze ans " et, encore, n'avait-il «jamais cru bien fort " en Dieu, mais "athée par orgueil», il reconnut souvent avoir gardé la foi, une certaine foi invincible et longtemps ininterrogée : une "foi en [s]on destin ", d'où lui sont venues sa "passion maniaque d'écrire » et la garantie de sa gloire future :

Cette façon d'être embarqué, dès l'enfance et sans avoir pu réfléchir, dans une "grande vie" comme d'autres dans la foi catholique ou communiste, m'a toujours interdit les inquiétudes et les crises de conscience où je voyais se complaire tant de mes camarades. J'étais assuré, j'avais la foi du charbonnier. [...] Ma vie, c'était une entreprise. Mais une entreprise favorisée des dieux ${ }^{19}$.

21 Et ce mandat sans mandant, qui lui a été donné en structurant sa vie une fois pour toutes, devait maintenir en lui, comme l'écrit Jacques Derrida, «l'unité métaphysique de l'homme et de Dieu, le rapport de l'homme à Dieu ». En cela, «l'athéisme ne change rien à cette structure fondamentale $»^{20}$, Dieu a moins été liquidé qu'il était passé chez Sartre tout entier dans cette incurable confiance en soi, dans son optimisme foncier, qui lui donnait l'assurance du présent, puisque celui-ci ne faisait rien d'autre que façonner l'essence en soi du grand écrivain qu'il devait être : «En somme j'ai l'obscure et profonde certitude d'avoir atteint par nature à un degré de perfection morale qu'il s'agit seulement de mériter ensuite par mes actes ${ }^{21}$.» C'est ainsi que la morale qu'il cherchait et qui avait défini son projet d'existence reposait depuis le début sur un fonds d'inauthenticité ; elle n'était que le moyen qu'il avait trouvé de faire son propre salut.

Autrement dit, la haute lucidité acquise par Sartre lui avait longtemps caché l'aveuglement sur soi qui lui avait permis de la conquérir : « [...] Truqué jusqu'à l'os et mystifié, j'écrivais joyeusement sur notre malheureuse condition. Dogmatique je doutais de tout sauf d'être l'élu du doute $\aleph^{22}$. Pour Sartre, tout avait été une comédie, une pièce jouée pour plaire à son grand-père; autour de lui, tout devait être une comédie, et sa mission était d'en porter témoignage aux autres et de les démystifier par l'écriture. Il avait cru que le christianisme n'avait eu aucun effet sur sa nature irreligieuse, sans voir que mort comme religion, il subsistait comme culture et qu'en celle-ci, il avait forgé ses propres notions pour se comprendre lui-même. Bref, lui, Sartre avait poussé, « herbe folle, sur le terreau de la catholicité ». À douze ans, il avait 
quitté Dieu, mais Dieu, à travers "la foi des autres", ne l'avait pas quitté. En le débaptisant, Sartre n'avait fait que le rendre invisible, et Dieu, absent de la lettre, devait demeurer présent en esprit, « installé à l'arrière de [s]a tête » : il était le "SaintEsprit, celui qui garantissait [s]on mandat et régentait [s]a vie». C'est ainsi que l'adolescent, acquis définitivement à l'absence de Dieu, avait laissé dans l'ombre l'enfant qui lui avait déjà, par avance, façonné son projet d'existence : « Ma seule affaire était de me sauver [...] par le travail et la foi $»^{23}$.

\section{La foi impossible que nous avons reçue du $x x^{e}$ siècle}

Le $\mathrm{xx}^{\mathrm{e}}$ siècle avait donné à l'universalité singulière de sa foi, et de son doute, une forme bien trop paradoxale pour durer très longtemps et nous autres, aujourd'hui, sommes dans l'impossibilité de nous y reconnaître. Même la mauvaise foi, qui assurait à la foi de se maintenir à travers des déguisements, nous est interdite. Car elle supposait encore la foi qu'elle pénétrait par insinuation, quand bien même ce n'était qu'afin de la ronger intérieurement. Sans la foi qu'elle abuse, elle-même est vouée à disparaitre. Du moins, il devient difficile de s'y complaire. Et ce que nous avons reçu de Sartre, et du siècle qu'il représente, est plutôt la lucidité crûment jetée sur notre propre situation.

Nous ne savons plus croire, car nous ne bénéficions plus des forces anonymes de la société qui transmettaient à chacun la foi sous sa forme implicite ou voilée. La religion n'est plus même une culture. En 1962, Joseph Masson avait forgé le mot d'« inculturation », que l'Église adopta à sa suite en vue de favoriser le dialogue entre la foi chrétienne et la diversité des cultures humaines. C'est ce christianisme inculturé qui expliquerait qu'au $\mathrm{XX}^{\mathrm{e}}$ siècle l'individu ait parfois retrouvé la foi après l'avoir perdue, qu'il ait pu la conserver en la dévoyant sur les chemins du politique. En 2003, Danielle Hervieu-Leger dut forger celui d'« exculturation $»^{24}$ pour signifier le divorce entre la religion et la culture qu'elle avait produite et dans laquelle elle se recréait elle-même. La religion s'était diluée dans la société ; elle en est désormais exclue. Elle a perdu « son ancrage culturel, et la culture a oublié ses sources religieuses et tout savoir profane du religieux $»^{25}$.

Autrement dit, la foi ne semble devoir compter à présent que sur nos seules forces. Coupée dans son élan, privée de ses anciens ancrages dans la société, elle ne peut relever aujourd'hui que d'une décision. Et c'est ce qui la rend impossible pour nous. Il ne s'agit pas de contester la nécessité de faire acte de foi ou que la volonté lui appartienne comme un de ses éléments essentiels, fût-ce pour consentir à la foi reçue et lui demeurer fidèle. Mais y suspendre la foi tout entière, comme le prétend Sartre, ne peut qu'entraver dès sa naissance l'impulsion première et immédiate qui nous en fournirait ne serait-ce que le désir. Car il est vrai que la conscience empoisonne tout et que la réflexion, en nous détachant des croyances, empêche d'adhérer pleinement à leurs contenus. Croit-on soudainement qu'on croit qu'un doute s'installe au fond de notre croyance : cette croyance que j'ai, ce n'est qu'une croyance, ma croyance. D'où l'oscillation entre les deux sens du verbe croire, le sens fort et le sens faible, puisqu'il indique aussi bien l'inébranlable fermeté de la foi (“je crois en vous, mon Dieu”) que le doute (“je n'en sais rien, je le crois"). Dès lors, une foi sous haute surveillance, placée dans la nécessité d'être explicite pour ne pas être nulle, est déjà une foi annulée : « La croyance, en devenant croyance pour soi, passe à l'état de non-croyance ${ }^{26}$. Comment la foi pourrait-elle encore gouverner notre conduite, éclairer le monde et orienter le sens 
de nos actions si, au lieu de nous soutenir ou d'exiger de s'y tenir, il nous faut la tenir, l'entretenir et la retenir sans cesse? C'est cette lucidité aujourd'hui requise qui empêche de s'éveiller à la foi aussi bien que de s'endormir dans la mauvaise foi. Car celle-ci, en mobilisant la réflexion, ne peut être longtemps cachée à celui qui voudrait se contenter de son peu de foi. La comédie du croire ne peut que tourner court et n'a plus même la stabilité de son instabilité. Elle ressemblerait assez à celle que Sartre disait avoir été celle de Merleau-Ponty : un jour qu'il passait à l'abbaye de Solesme pour y communier, celui-ci «crut voir se reformer en face de lui, contre lui cette totalité dont il avait la nostalgie ; il imagina ce retour à l'enfance : l'enveloppement par la foi. Il se convertit, essaya de croire, ce fut un détour de sa méditation sur soi ; il conclut, au bout de quelques années : «On croit qu'on croit mais on ne croit pas $»^{27}$.

Après la seconde grande guerre, la sociologie de la religion pouvait encore suivre la fin du processus de déchristianisation en Europe jusqu'à son étiage le plus bas et, sur sa large palette, distinguer avec finesse les croyants pratiquants, hebdomadaires ou occasionnels, les croyants non pratiquants ou les pratiquants non croyants, enfin les non-croyants attachés culturellement à leur religion; aujourd'hui, ces catégories s'abîment dans l'indistinction d'une croyance problématique qui s'enlève sur un fond d'incrédulité généralisée. Nous ne savons plus croire. Nous croyons peut-être croire, au mieux nous vivons dans la nostalgie de l'ancienne foi et prenons volontiers notre désir de croire pour la croyance elle-même. Pas davantage ne savons-nous douter vraiment et redescendre jusqu'aux raisons critiques qui avaient su nous armer contre une religion jadis intrusive. Car nous ne sommes pas tant athées que les fruits de l'athéisme, lequel a accompli son œuvre et s'est édulcoré en même temps que s'est affaibli l'adversaire qu'il s'était choisi. L'athée était, il y a peu encore, un croyant, en creux et en négatif, par la fougue même avec laquelle il se jetait dans le combat. Et Auguste Comte rangeait l'athéisme dans l'âge métaphysique de l'humanité, que l'esprit positif et scientifique devait laisser derrière lui, en cessant d'éprouver le besoin d'aller en quête de ce qu'il savait ne pouvoir savoir. En cela l'athée véritable comme Sartre l'a résolument été est de plus en plus rare. Et sa figure s'est elle-même édulcorée en se rapprochant étrangement de la figure du croyant à mesure que celle-ci devait être ramenée vers elle à qui elle avait soufflé ses propres doutes.

\section{NOTES}

1. H. de Lubac, La Postérité spirituelle de Joachim de Flore, Paris, Éditions du Cerf, 2014, p. 838.

2. J.-P. Sartre, Carnets de la drôle de guerre, III, dans Les Mots et autres écrits autobiographiques, Paris, Gallimard, «Bibliothèque de la Pléiade », 2014, p. 353.

3. J.-P. Sartre, «L'universel singulier », Situations, IX, Paris, Gallimard, 1972, p. 172.

4. A. Gide, Journal, 13 décembre 1927, Paris, Gallimard, « Bibliothèque de la Pléiade », vol. II, 1997, p. 62.

5. J.-P. Sartre, L'Idiot de la famille (1971), Tome I, Paris, Gallimard, 1988, p. 7.

6. J.-P. Sartre, L'Être et le Néant (1943), Paris, Gallimard, coll. « Tel », 2009, I, p. 104 et 105.

7. Ibid., p. 103. 
8. Ibid., p. 105, 111 et 105 .

9. M. Merleau-Ponty, «Foi et bonne foi » (1946), dans Sens et non-sens, Paris, Gallimard, 1996, p. 217.

10. J.-P. Sartre, L'Être et le Néant, op. cit., p. 106, note 1.

11. J.-P. Sartre, Carnets de la drôle de guerre, III, dans Les Mots et autres écrits autobiographiques, p. 331 et 334 .

12. J.-P. Sartre, Mallarmé, la lucidité et sa face d'ombre, Paris, Gallimard, coll. « Arcades », 1986, p. 17.

13. Ibid., p. 18.

14. J.-P. Sartre, L'Être et le Néant, op. cit., p. 84.

15. J.-P. Sartre, Cahier Lutèce, dans Les Mots et autres écrits autobiographiques, op. cit., p. 925.

16. J.-P. Sartre, Mallarmé, La lucidité et sa face d'ombre, op. cit., p. 19.

17. Épître de saint Jacques, 2, 19, Bible de Jérusalem, Paris, Les éditions du Cerf, 1998, p. 2031 ; voir F. Hadjadj, La Foi des démons ou l'athéisme dépassé, Paris, Albin Michel, 2011.

18. C. Péguy, Clio, Dialogue de l'histoire et de l'âme païenne (1913), œuvres posthumes, édition présentée, établie et annotée par R. Burac, Paris, Gallimard, «Bibliothèque de la Pléiade », III, 1992 , p. 1129.

19. J.-P. Sartre, Carnets de la drôle de guerre, dans Les Mots et autres écrits autobiographiques, op. cit., III, p. 352 ; XIV, 616, 632 ; III, p. 361.

20. J. Derrida, Marges de la philosophie (1972), Paris, Éditions de Minuit, 1997, p. 138.

21. J.-P. Sartre, Carnets de la drôle de guerre, op. cit., I, p. 237.

22. J.-P. Sartre, Les Mots, dans Les Mots et autres écrits autobiographiques, op. cit., p. 137.

23. Ibid., p. 135, 136 et 139 .

24. D. Hervieu-Léger, Catholicisme, la fin d'un monde, Paris, Bayard, 2003.

25. O. Roy, La Sainte Ignorance, Paris, Seuil, coll. « Points Essais », 2012, p. 209.

26. J.-P. Sartre, L'Être et le Néant, op. cit., p. 104.

27. J.-P. Sartre, Merleau-Ponty (Première version, manuscrite), dans Les Mots et autres écrits autobiographiques, op. cit., p. 1130. 